\title{
Temperature dependent charge-injection at the metal-organic semiconductor interface and density of states in pristine and doped pentacene
}

\author{
Claudio Vanoni, Thomas A. Jung, ${ }^{\text {a) }}$ and Soichiro Tsujino ${ }^{\text {b) }}$ \\ Laboratory for Micro- and Nanotechnology, Paul Scherrer Institut, CH-5232 Villigen-PSI, Switzerland
}

(Received 24 March 2009; accepted 7 June 2009; published online 25 June 2009)

\begin{abstract}
The authors study temperature dependent electrical transport in pristine and tetrafluorotetracyanoquinodimethane $\left(\mathrm{F}_{4} \mathrm{TCNQ}\right)$-doped pentacene to explore the physical mechanism of the doping-induced reduction in the contact resistance at the metal-organic semiconductor interface. It was found that the $\mathrm{F}_{4}$ TCNQ-doping induces an apparent lowering of the hole injection barrier at gold-pentacene interfaces. In addition, characteristic changes in the density of states (DOS) induced by the $\mathrm{F}_{4} \mathrm{TCNQ}$-doping in a thin-film transistor geometry have been observed. The correlation between the doping-induced DOS and the carrier injection process is discussed. () 2009 American Institute of Physics. [DOI: 10.1063/1.3159835]
\end{abstract}

Charge-injection at semiconductor-contact interfaces is one of the performance limiting factors of organic semiconductor devices. Doping has been known to improve chargeinjection properties in inorganic, as well as in organic semiconductors. ${ }^{1}$ In particular, the doping of pentacene (PC) thin films by tetrafluorotetracyanoquinodimethane ( $\mathrm{F}_{4} \mathrm{TCNQ}$ ) can reduce the contact resistance $R_{c}$ between gold and $\mathrm{PC} .^{2,3}$ In few-monolayer (ML) PC thin-film transistors (TFTs), we observed recently a factor of 20 reduction of $R_{c}$ at room temperature by a few percent doping of $\mathrm{F}_{4} \mathrm{TCNQ}^{2}{ }^{2}$ However, the physical mechanism of the doping-induced $R_{c}$ reduction has remained elusive. In the past, the decrease in the depletion layer thickness by doping and concomitant increase in tunneling injection through the depletion barrier have been suggested in analogy to mechanisms described for inorganic semiconductors. ${ }^{4,5}$ Doping at contacting interfaces has been shown to affect the alignment between the Fermi level of contacting metal and molecular levels. ${ }^{6-8}$ Blochwitz et $a .^{7}{ }^{7}$ studied the energy-level alignment at the interfaces between metal films (gold and indium-tin-oxide) and thick zinc-phthalocynanine films and found a reduction in holeinjection barrier height in the order of $0.1-0.2 \mathrm{eV}$ upon $\mathrm{F}_{4}$ TCNQ-doping. Koch et al. ${ }^{8}$ demonstrated that electron transfer from $\mathrm{Au}$ to $\mathrm{F}_{4} \mathrm{TCNQ}$ at the interface is responsible for the observed lowering of the hole-injection barrier by $1.2 \mathrm{eV}$. The influence of the density of states (DOS) and the impact of the doping-induced disorder of DOS on the charge-injection have been proposed, ${ }^{9-11}$ and the DOS in pristine and doped organic semiconductors have been reported. $^{12-14}$ However, the importance of doping-induced disorder on the barrier height reduction ${ }^{10,11}$ and the correlation of the experimentally determined DOS with $R_{c}$ are still not apparent from these studies.

In this letter, we approach this question by studying the temperature dependence of the electrical transport in doped and pristine PC nanocrystals and in few MLs-thick PC TFTs. These experiments allow for the assessment of the DOS of pristine and $\mathrm{F}_{4}$ TCNQ-doped PCs in TFT geometries. Our

\footnotetext{
${ }^{a)}$ Electronic mail: thomas.jung@psi.ch.

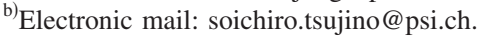

results indicate that $R_{c}$-reduction is caused by specific interactions between the guest molecule ( $\left.\mathrm{F}_{4} \mathrm{TCNQ}\right)$ and the host molecular PC film.

The fabrication procedure of TFT and nanocrystal samples was described previously (Refs. 2 and 15). The PC nanocrystals, (0.2-1) $\mu \mathrm{m}$ length and (10-200) $\mathrm{nm}$ width, were self-assembled on $\mathrm{Au}$ nanojunctions with $\sim 10 \mathrm{~nm}$ electrode separation. TFTs were fabricated by depositing a (6-7) ML-thick PC channel on a $p^{+}$-doped oxidized silicon substrate prepatterned with $\mathrm{Au}$ electrodes. The thickness of the oxide was $150 \mathrm{~nm}$. Prior to PC deposition experiments, the nanojunctions and TFT chips were exposed to an $\mathrm{O}_{2}$-plasma for cleaning by using a reactive ion etcher (Oxford Plasmalab) for $30 \mathrm{~s}$ at $100 \mathrm{~W}$. Four TFTs with different contact separations $L$ between 20 and $200 \mu \mathrm{m}$ were fabricated at the same time for the purpose of evaluating the channel-length dependence of the TFT characteristics and the contact resistance. The doped PC was deposited by evaporation of PC mixed with $\mathrm{F}_{4}$ TCNQ (Refs. 2 and 15). The concentration of $\mathrm{F}_{4} \mathrm{TCNQ}$ was estimated to be $1.5 \% \pm 0.5 \%$ for TFTs and $3 \% \pm 1 \%$ for nanocrystals. The room temperature transport measurements were performed in situ in the vacuum. For the temperature dependent transport measurements, fabricated devices were transferred to a cryostat while limiting the air exposure to less than $5 \mathrm{~min}$. The cryostat was evacuated to a pressure below $10^{-6}$ Torr.

Using TFTs with a 200- $\mu \mathrm{m}$-long and 110- $\mu \mathrm{m}$-wide channel, we also measured the DOS of the PC films, $D(E)$, by applying the method reported by Lang et al. ${ }^{12}$ and Vanoni: ${ }^{15}$ from the relation between the gate bias voltage $V_{g}$ and the activation energy $E_{a}^{(G)}$ of the channel conductance $G_{\mathrm{ds}}, D(E)$ is evaluated as,

$$
D(E)=1 /\left(q C_{\mathrm{ox}} t_{\mathrm{ch}}\right) \times\left.\left[\partial E_{a}^{(G)} / \partial V_{g}\right]^{-1}\right|_{E_{a}^{(G)}=E},
$$

where the origin of the energy scale is set to the highest occupied molecular orbital (HOMO) level of PC, $t_{\mathrm{ch}}$ is the channel thickness equal to $10 \mathrm{~nm}, C_{\mathrm{ox}}$ is the oxide capacitance equal to $2.4 \times 10^{-8} \mathrm{~F} \mathrm{~cm}^{-2}$, and $q$ is the elementary charge. Following Lang et al., ${ }^{12}$ we assume that (i) most of the carriers are trapped in the gap states between HOMO and the lowest unoccupied molecular orbital states and do not 
(a)

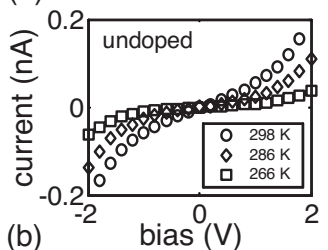

(b)

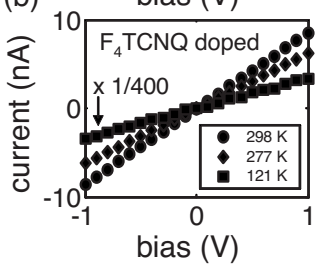

(c)

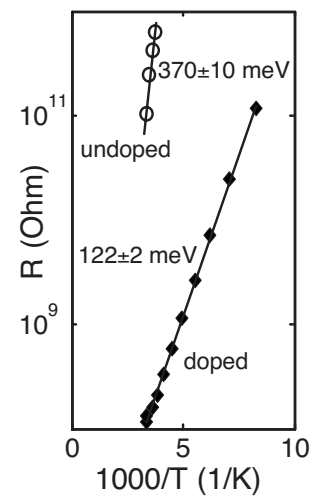

FIG. 1. Temperature dependent $I-V$ characteristics of pristine (a) and $3 \%$ - F $_{4}$ TCNQ-doped (b) PC nanocrystals self-assembled on Aunanojunctions with $\sim 10 \mathrm{~nm}$ electrode separation/channel length. Doping increases the current by a factor $\sim 100$ and changes the rectifying behavior to linear $I-V$ down to $121 \mathrm{~K}$. The panel (c) shows the Arrhenius plot of zero-bias resistance for pristine (empty circle) and doped PC nanocrystals (solid diamonds).

contribute to $G_{\mathrm{ds}}$; (ii) consequently $G_{\mathrm{ds}}$ is proportional to the carrier concentration thermally excited from the states below the thermal energy, $k_{B} T$; (iii) the temperature dependence of the mobility of the free carriers in the transport level can be neglected. We found that (i) and (ii) are consistent with the evaluated DOS since $E_{a}^{(G)}$ and the relevant energy range is much larger than $k_{B} T$ in our experiment. (iii) is justified by the results obtained by techniques that measure the intrinsic carrier mobility. ${ }^{16-18}$ In fact, it was shown that the activation energy of the carrier mobility is smaller than the activation energy of $G_{\mathrm{ds}}$ observed here. In order to evaluate the derivative in Eq. (1) numerically, the relation between $E_{a}^{(G)}$ and $V_{g}$ is smoothened by a piecewise linear fit of 7-15 points, which introduces an energy resolution of $\sim 11 \mathrm{meV}$ (Fig. 3).

In Figs. 1(a) and 1(b), we show the two-terminal currentvoltage characteristics $(I-V)$ of pristine and $\mathrm{F}_{4} \mathrm{TCNQ}$-doped PC nanocrystals at various temperatures between 300 and $121 \mathrm{~K}$. The pristine PC exhibits a rectifying $I-V$ indicating that holes are injected by thermionic emission. This interpretation is consistent with the rapid increase in the zero bias resistance with the decrease in temperature characterized by the activation energy $E_{a}^{(\text {ud) }}$ equal to $370 \pm 10 \mathrm{meV}$ [Fig. $1(\mathrm{c})] . E_{a}^{(\mathrm{ud})}$ is dominated by the contact resistance for undoped PC TFTs with channel lengths below $20 \mu \mathrm{m}$. $^{2}$ Therefore we tentatively ascribe $E_{a}^{(\mathrm{ud})}$ to the barrier height at the contact interface.

In contrast, the comparison of Figs. 1(a) and 1(b) at $298 \mathrm{~K}$ shows that $3 \%$ doping of $\mathrm{F}_{4}$ TCNQ to PC resulted in the decrease in the zero bias resistance by a factor $\lambda_{\exp }$ of $10^{3}$ and the increase in the current at the bias of $1 \mathrm{~V}$ by a factor 500. The resistance of the $\mathrm{F}_{4}$ TCNQ-doped PC nanocrystal increases with the decrease in the temperature with an activation energy $E_{a}^{(d)}$ equal to $122 \pm 2 \mathrm{meV}$ (Fig. 1). This result suggests that the $\mathrm{F}_{4} \mathrm{TCNQ}$-doping reduces the contact resistance by lowering the barrier height for the hole injection at the gold-PC interface. This is also compatible with the observed ratio $\lambda_{\text {exp }}$. According to the Richardson-Schottky thermionic injection mechanism, the zero bias resistance ratio $\lambda_{\mathrm{TE}}$ is written as $A \exp \left(\Delta \Phi / k_{B} T\right)$, where $\Delta \Phi$ is the decrease in the injection barrier height and $A$ is the ratio of the cross section of the transport layers. Using the experimen-

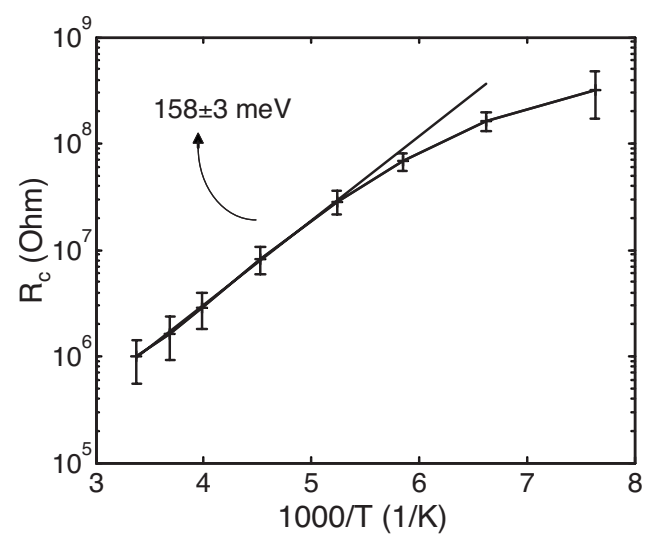

FIG. 2. Contact resistance $R_{c}$ vs $1000 / T$ evaluated by the transmission line method applied to $\mathrm{F}_{4}$ TCNQ doped PC TFTs. Above $190 \mathrm{~K}, R_{c}$ shows an Arrhenius behavior with a thermal activation energy $E_{a}^{\left(R_{c}\right)}$ of $158 \pm 3 \mathrm{meV}$.

tally determined values, $\Delta \Phi$ of $\sim 0.25 \mathrm{eV}$ given by $\left[E_{a}^{(\mathrm{ud})}-E_{a}^{(d)}\right]$ and $A$ of $\sim 0.3$ (Ref. 19), $\lambda_{\mathrm{TE}}$ equal to $\sim 5 \times 10^{3}$ is evaluated that is of the same order of magnitude as $\lambda_{\text {exp }}$.

The apparent lowering of the barrier height at the interface between gold and $\mathrm{F}_{4}$ TCNQ-doped PC was also observed in TFT geometry. Figure 2 shows the relation between $R_{c}$ and inverse temperatures observed for the $\mathrm{F}_{4}$ TCNQ-doped PC TFTs. The temperature dependence of $R_{c}$ above $190 \mathrm{~K}$ is represented by an activation energy $E_{a}^{\left(R_{c}\right)}$ equal to $158 \pm 3 \mathrm{meV}$. This value agrees to $E_{a}^{(d)}$ observed above for the doped-PC-nanocrystal within $30 \%$. This difference is associated to a factor of 2 higher doping concentration in nanocrystals. The deviation from Arrhenius behavior of $R_{c}$ below $190 \mathrm{~K}$ can be ascribed to the hopping-limited diffusion mechanism. ${ }^{20}$ In the case of pristine PC exposed to the air, the evaluation of $R_{c}$ was difficult since $R_{\mathrm{tot}}$ did not vary linearly with $L$. However, we found that the temperature dependence of $G_{\mathrm{ds}}$ in undoped PC TFTs with $20 \mu \mathrm{m}$-long channel, when extrapolated to gate voltages $V_{g}$ equal to $0 \mathrm{~V}$, is consistent with $E_{a}^{\text {(ud) } 21}$.

Finally, to study the influence of the doping-induced disorder, we measured the DOS in pristine PC (open circles) and $\mathrm{F}_{4}$ TCNQ-PC (filled squares), Fig. 3. The DOS of pristine PC agrees to the DOS reported in the literature ${ }^{12,15,22,23}$ within the measured energy range. In contrast, the $\mathrm{F}_{4}$ TCNQ-doped PC exhibits an additional DOS peak cen-

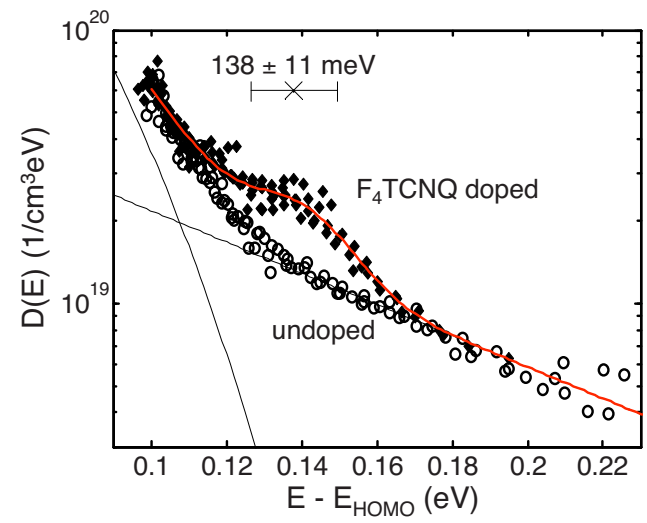

FIG. 3. (Color online) DOS $D(E)$ in undoped (empty circles) and $\mathrm{F}_{4}$ TCNQ doped (solid diamonds) PC TFTs. The center of the HOMO transport level is chosen as the origin of the energy $E$. The straight lines indicate the fit of the shallow and deep region of the pristine PC. 
tered at $0.138 \mathrm{eV}$ above the HOMO level. We ascribe this peak as the acceptor states created by the $\mathrm{F}_{4}$ TCNQ molecules. The total area $p_{\text {dos }}$ of the peak is equal to $(3 \pm 1)$ $\times 10^{17} \mathrm{~cm}^{-3}$, which is in good agreement with the concentration $p_{\text {ac }}$ of the activated $\mathrm{F}_{4}$ TCNQ molecules equal to $\sim 1$ $\times 10^{17} \mathrm{~cm}^{-3}$ (Refs. 15 and 24). However, we do not observe apparent disorder in the doped PC. In fact, away from the peak, the DOS shows similar values for pristine and doped PC. In addition, we found that the mobility values of the pristine and $\mathrm{F}_{4}$ TCNQ-doped TFTs measured in situ and ex situ (in the cryostat) were found to be both equal to $(0.3-0.4) \mathrm{cm}^{2} /(\mathrm{V} \mathrm{s})$; no degradation of the PC film quality was observed either by the $\mathrm{F}_{4}$ TCNQ-doping or by the brief exposure of the TFT to the air during the sample transfer. It should also be noted that the influence of the contact on the extracted DOS is minimal when the DOS is evaluated using devices with $L$ of $200 \mu \mathrm{m}$. In fact, the same analysis for shorter channel devices down to $20 \mu \mathrm{m}$ showed that, the $V_{g}$ dependence of $E_{a}^{(G)}$ decreases systematically when $L$ is decreased, and that the DOS peak induced by the acceptor states is reduced. ${ }^{25}$

The here observed similarity between the activation energy of $R_{c}$ in doped PC and the doping induced acceptor states suggests that the origin of the increase in the injection current lies in the increased DOS at the injection energy. This effect is a result of a specific interaction between PC and the dopant molecules rather than a disorder induced mechanism. The three to four times higher activation energies of $R_{c}$ in undoped PC clearly show that the injection in doped PC is governed by different mechanisms.

In conclusion, we have shown that doping $\mathrm{PC}$ with the strong electron acceptor molecules $\mathrm{F}_{4}$ TCNQ decreases the contact resistance and produces additional states. The temperature dependence of $\mathrm{F}_{4}$ TCNQ-doped PCs in TFT devices and $10 \mathrm{~nm}$ channel devices indicates that the reduction in the barrier height at the metal-semi-interface induced by the doping is the origin of the doping-induced contact resistance reduction. While the molecular scale mechanism of such barrier height reduction needs to be clarified by further microscopic investigations, our results demonstrate that further improvement of $R_{c}$ is possible by optimization of doping species and doping methods.

We acknowledge R. Schelldorfer for technical support in constructing the experimental setup and in the pentacene deposition, and N. Baumann and C. Schönenberger for interesting and valuable discussions. This work was partially supported by the Swiss National Center (NCCR) on "Nanoscale Science" and by the Swiss National Science Foundation (SNSF).
${ }^{1}$ Y. Shen, A. R. Hosseini, M. H. Wong, and G. G. Malliaras, ChemPhysChem 5, 16 (2004).

${ }^{2}$ C. Vanoni, S. Tsujino, and T. A. Jung, Appl. Phys. Lett. 90, 193119 (2007).

${ }^{3}$ T. Minari, T. Miyadera, K. Tsukagoshi, Y. Aoyagi, and H. Ito, Appl. Phys. Lett. 91, 053508 (2007).

${ }^{4}$ W. Gao and A. Kahn, Org. Electron. 3, 53 (2002).

${ }^{5}$ M. Pfeiffer, K. Leo, X. Zhou, J. S. Huang, M. Hofmann, A. Werner, and J. Blochwitz-Nimoth, Org. Electron. 4, 89 (2003).

${ }^{6}$ B. H. Hamadani, H. Ding, Y. Gao, and D. Natelson, Phys. Rev. B 72 , 235302 (2005).

${ }^{7}$ J. Blochwitz, T. Fritz, M. Pfeiffer, K. Leo, D. M. Alloway, P. A. Lee, and N. R. Armstrong, Org. Electron. 2, 97 (2001).

${ }^{8}$ N. Koch, S. Duhm, J. P. Rabe, A. Vollmer, and R. L. Johnson, Phys. Rev. Lett. 95, 237601 (2005).

${ }^{9}$ A. R. Volkel, R. A. Street, and D. Knipp, Phys. Rev. B 66, 195336 (2002).

${ }^{10}$ A. R. Hosseini, M. H. Wong, Y. Shen, and G. G. Malliaras, J. Appl. Phys. 97, 023705 (2005).

${ }^{11}$ V. I. Arkhipov, P. Heremans, E. V. Emelianova, and H. Bässler, Phys. Rev. B 71, 045214 (2005).

${ }^{12}$ D. V. Lang, X. Chi, T. Siegrist, A. M. Sergent, and A. P. Ramirez, Phys. Rev. Lett. 93, 086802 (2004).

${ }^{13}$ I. N. Hulea, H. B. Brom, A. J. Houtepen, D. Vanmaekelbergh, J. J. Kelly, and E. A. Meulenkamp, Phys. Rev. Lett. 93, 166601 (2004).

${ }^{14}$ O. Tal, Y. Rosenwaks, Y. Preezant, N. Tessler, C. K. Chan, and A. Kahn, Phys. Rev. Lett. 95, 256405 (2005).

${ }^{15} \mathrm{C}$. Vanoni, Thesis, University of Basel, 2007.

${ }^{16}$ V. Podzorov, E. Menard, J. A. Rogers, and M. E. Gershenson, Phys. Rev. Lett. 95, 226601 (2005).

${ }^{17}$ R. W. I. de Boer, M. Jochemsen, T. M. Klapwijk, A. F. Morpurgo, J. Niemax, A. K. Tripathi, and J. Pflaum, J. Appl. Phys. 95, 1196 (2004)

${ }^{18}$ T. Minari, T. Nemoto, and S. Isoda, J. Appl. Phys. 99, 034506 (2006).

${ }^{19}$ The ratio $A$ was evaluated from the ratio of the deposited thickness $(6 \mathrm{ML}$ for the doped and $20 \mathrm{ML}$ for the undoped PC nanocrystal) equal to $\sim 0.3$ and the ratio of the effective PC channel width of $\sim 1$ observed by SEM of actual nanojunction, see Ref. 15 .

${ }^{20}$ U. Wolf, V. I. Arkhipov, and H. Bässler, Phys. Rev. B 59, 7507 (1999).

${ }^{21}$ We ascribe the difficulty to apply TLM method to undoped PC TFTs to the large non-Ohmic contact properties and its considerable influence on the short channel devices at low temperatures.

${ }^{22}$ R. Coehoorn, W. F. Pasveer, P. A. Bobbert, and M. A. J. Michels, Phys. Rev. B 72, 155206 (2005).

${ }^{23}$ M. C. J. M. Vissenberg and M. Matters, Phys. Rev. B 57, 12964 (1998).

${ }^{24}$ Here, $p_{\text {ac }}$ was evaluated from the positive threshold gate voltage shift of $\sim 1 \mathrm{~V}$ of the doped-PC TFT compared to the pristine-PC TFT at room temperature. The total concentration of the $\mathrm{F}_{4} \mathrm{TCNQ}$ molecules codeposited in the PC film is equal to $\sim 4 \times 10^{19} \mathrm{~cm}^{-3}$. Therefore, the fraction of the electronically active $\mathrm{F}_{4} \mathrm{TCNQ}$ is in the order of $10^{-2}$. Previous estimates of the doping efficiency of $\mathrm{F}_{4} \mathrm{TCNQ}$ molecule to PC returned a value equal to $\sim 0.1$ holes/dopant in vacuum. The lower doping efficiency by a factor of about 10 observed here, is ascribed to the brief ( $\sim \mathrm{min})$ exposure of the sample to the ambient air during the transfer from the PC deposition chamber to the cryostat (see also Ref. 15).

${ }^{25}$ The doping-induced states observed here is potentially consistent with the previously observed $V_{g}$-dependence of $R_{c}$ in TFTs (Ref. 2); An increase of $R_{c}$ upon increasing the negative $V_{g}$ above $10 \mathrm{~V}$ can be ascribed to the resonantlike-injection from $\mathrm{Au}$ into the doping-induced states detuned by $V_{g}$. 